\title{
Level-Spacing Distributions and the Airy Kernel
}

\author{
Craig A. Tracy円 \\ Department of Mathematics and Institute of Theoretical Dynamics, \\ University of California, Davis, CA 95616, USA \\ Harold Widomi \\ Department of Mathematics, \\ University of California, Santa Cruz, CA 95064, USA
}

\begin{abstract}
Scaling level-spacing distribution functions in the "bulk of the spectrum" in random matrix models of $N \times N$ hermitian matrices and then going to the limit $N \rightarrow \infty$, leads to the Fredholm determinant of the sine kernel $\sin \pi(x-y) / \pi(x-y)$. Similarly a scaling limit at the "edge of the spectrum" leads to the Airy kernel $\left[\operatorname{Ai}(x) \operatorname{Ai}^{\prime}(y)-\operatorname{Ai}^{\prime}(x) \operatorname{Ai}(y)\right] /(x-y)$. In this paper we derive analogues for this Airy kernel of the following properties of the sine kernel: the completely integrable system of P.D.E.'s found by Jimbo, Miwa, Môri and Sato; the expression, in the case of a single interval, of the Fredholm determinant in terms of a Painlevé transcendent; the existence of a commuting differential operator; and the fact that this operator can be used in the derivation of asymptotics, for general $n$, of the probability that an interval contains precisely $n$ eigenvalues.
\end{abstract}

\footnotetext{
*e-mail address: catracy@ucdavis.edu

†e-mail address: widom@cats.ucsc.edu
} 


\section{INTRODUCTION AND SUMMARY OF RESULTS}

\section{A. Introduction}

In this paper we present new results for the level spacing distribution functions obtained from scaling random matrix models of $N \times N$ hermitian matrices at the edge of the support of the (tree-level) eigenvalue densities when the parameters of the potential $V$ are not "finely tuned." This universality class is already present in the Gaussian Unitary Ensemble. It is known [3, 10,20,23] that these distribution functions are expressible in terms of a Fredholm determinant of an integral operator $K$ whose kernel involves Airy functions.

It turns out that there are striking analogies between the properties of this Airy kernel

$$
K(x, y)=\frac{A(x) A^{\prime}(y)-A^{\prime}(x) A(y)}{x-y}
$$

where $A(x)=\sqrt{\lambda} \operatorname{Ai}(x)$, and the sine kernel

$$
\frac{\lambda}{\pi} \frac{\sin \pi(x-y)}{x-y}
$$

whose associated Fredholm determinant describes the classical level spacing distribution functions first studied by Wigner, Dyson, Mehta, and others [0],20,26].

To be a little more explicit about how these kernels arise we remind the reader that in the GUE the probability density that the eigenvalues of a random $N \times N$ hermitian matrix lie in infinitesimal intervals about $x_{1}, \cdots, x_{N}$ is given by 20]

$$
P_{N}\left(x_{1}, \cdots, x_{N}\right)=\frac{1}{N !} \operatorname{det}\left(K_{N}\left(x_{i}, x_{j}\right)\right)_{i, j=1, \cdots, N}
$$

where

$$
K_{N}(x, y)=\sum_{k=0}^{N-1} \varphi_{k}(x) \varphi_{k}(y)
$$

Here $\left\{\varphi_{k}(x)\right\}$ is the sequence obtained by orthonormalizing the sequence

$$
\left\{x^{k} \exp \left(-x^{2}\right)\right\}
$$


over $(-\infty, \infty)$. More generally the $n$-point correlation function $R_{n}\left(x_{1}, \cdots, x_{n}\right)$, which is the probability density that $n$ of the eigenvalues (irrespective of order) lie in infinitesimal intervals about $x_{1}, \cdots, x_{n}$, is given by

$$
R_{n}\left(x_{1}, \cdots, x_{n}\right)=\operatorname{det}\left(K_{N}\left(x_{i}, x_{j}\right)\right)_{i, j=1, \cdots, n}
$$

In particular $R_{1}(x)$, the density of eigenvalues at $x$, equals $K_{N}(x, x)$.

Replacing $K_{N}(x, y)$ by

$$
\frac{1}{R_{1}(z)} K_{N}\left(z+\frac{x}{R_{1}(z)}, z+\frac{y}{R_{1}(z)}\right)
$$

has the effect of making the point $z$ in the spectrum the new origin and rescaling so that the eigenvalue density at this point becomes equal to 1 . Now if $z$ is fixed then

$$
R_{1}(z)=K_{N}(z, z) \sim \frac{1}{\pi} \sqrt{2 N}
$$

as $N \rightarrow \infty$, and

$$
\lim _{N \rightarrow \infty} \frac{\pi}{\sqrt{2 N}} K_{N}\left(z+\frac{\pi x}{\sqrt{2 N}}, z+\frac{\pi y}{\sqrt{2 N}}\right)=\frac{1}{\pi} \frac{\sin \pi(x-y)}{(x-y)}
$$

so we obtain the sine kernel in this way. (This limit is obtained by applying the ChristoffelDarboux formula to $K_{N}(x, y)$ and then using the known asymptotics of $\varphi_{N}(x)$ as $N \rightarrow \infty$.) The "edge of the spectrum" corresponds to $z \sim \pm \sqrt{2 N}$ and one has there the scaling limit 10,23

$$
\lim _{N \rightarrow \infty} \frac{1}{2^{1 / 2} N^{1 / 6}} K_{N}\left(\sqrt{2 N}+\frac{x}{2^{1 / 2} N^{1 / 6}}, \sqrt{2 N}+\frac{y}{2^{1 / 2} N^{1 / 6}}\right)=\frac{\operatorname{Ai}(x) \operatorname{Ai}^{\prime}(y)-\operatorname{Ai}^{\prime}(x) \operatorname{Ai}(y)}{x-y} .
$$

We now present some of the analogies which we have found. (That such analogies between properties of the sine kernel and Airy kernel exist is perhaps not suprising since they are both scaling limits of the same family of kernels.) The first is the analogue of the completely integrable system of P.D.E.'s of Jimbo, Miwa, Môri, and Sato [17] when the underlying domain is a union of intervals. The second is the fact that in the case of the semi-infinite interval $(s, \infty)$ (the analogue of a single finite interval for the sine kernel) the 
Fredholm determinant is closely related to a Painlevé transcendent of the second kind (the fifth transcendent arises for the sine kernel [17]). And the third is the existence of a second order differential operator commuting with the Airy operator $K$. (The existence of such a differential operator in the sine kernel case has been known for some time, see e.g. [14], page 201.) This last fact leads to an explicit asymptotic formula, as the interval $(s, \infty)$ expands, for the probability that it contains precisely $n$ eigenvalues $(n=1,2, \ldots)$ (the analogue of results in [2,31]). Here are our results, stated in some detail. (A short announcement appears in 29. .)

\section{B. The System of Partial Differential Equations}

We set

$$
J=\bigcup_{j=1}^{m}\left(a_{2 j-1}, a_{2 j}\right)
$$

and write $D(J ; \lambda)$ for the Fredholm determinant of $K$ acting on $J$. We think of this as a function of $a=\left(a_{1}, \ldots, a_{2 m}\right)$. Then

$$
d_{a} \log D(J ; \lambda)=-\sum_{j=1}^{2 m}(-1)^{j} R\left(a_{j}, a_{j}\right) d a_{j}
$$

where $R(x, y)$ is the kernel of the operator $K(I-K)^{-1}$ and $d_{a}$ denotes exterior differentiation with respect to the $a_{j}$ 's. We introduce quantities

$$
q_{j}=(I-K)^{-1} A\left(a_{j}\right), \quad p_{j}=(I-K)^{-1} A^{\prime}\left(a_{j}\right),
$$

(which are the analogue of the quantities $r_{ \pm j}$ of [17]; see also [9, 15, 21, 22]) as well as two further quantities

$$
u=\left(A,(I-K)^{-1} A\right), \quad v=\left(A,(I-K)^{-1} A^{\prime}\right)
$$

where the inner products refer to the domain $J$. Then the equations read 


$$
\begin{aligned}
\frac{\partial q_{j}}{\partial a_{k}} & =(-1)^{k} \frac{q_{j} p_{k}-p_{j} q_{k}}{a_{j}-a_{k}} q_{k} \quad(j \neq k), \\
\frac{\partial p_{j}}{\partial a_{k}} & =(-1)^{k} \frac{q_{j} p_{k}-p_{j} q_{k}}{a_{j}-a_{k}} p_{k} \quad(j \neq k), \\
\frac{\partial q_{j}}{\partial a_{j}} & =-\sum_{k \neq j}(-1)^{k} \frac{q_{j} p_{k}-p_{j} q_{k}}{a_{j}-a_{k}} q_{k}+p_{j}-q_{j} u, \\
\frac{\partial p_{j}}{\partial a_{j}} & =-\sum_{k \neq j}(-1)^{k} \frac{q_{j} p_{k}-p_{j} q_{k}}{a_{j}-a_{k}} p_{k}+a_{j} q_{j}+p_{j} u-2 q_{j} v, \\
\frac{\partial u}{\partial a_{j}} & =(-1)^{j} q_{j}^{2}, \\
\frac{\partial v}{\partial a_{j}} & =(-1)^{j} p_{j} q_{j} .
\end{aligned}
$$

Moreover the quantities $R\left(a_{j}, a_{j}\right)$ appearing in (1.1) are given by

$$
R\left(a_{j}, a_{j}\right)=\sum_{k \neq j}(-1)^{k} \frac{\left(q_{j} p_{k}-p_{j} q_{k}\right)^{2}}{a_{j}-a_{k}}+p_{j}^{2}-a_{j} q_{j}^{2}-2 p_{j} q_{j} u+2 q_{j}^{2} v .
$$

These equations are derived very much in the spirit of [28]; see also [9, 15,21].

\section{The Ordinary Differential Equations}

For the special case $J=(s, \infty)$ the above equations can be used to show that $q(s ; \lambda)$ (the quantity $q$ of the last section corresponding to the end-point $s$ ) satisfies

$$
q^{\prime \prime}=s q+2 q^{3}, \quad\left({ }^{\prime}=\frac{d}{d s}\right)
$$

with

$$
q(s ; \lambda) \sim \sqrt{\lambda} \operatorname{Ai}(s) \quad \text { as } s \rightarrow \infty
$$

This equation is a special case of the $\mathrm{P}_{I I}$ differential equation [1, 16, 18, 25, One can similarly derive for $R(s):=R(s, s)$, which in view of (1.1) equals

$$
\frac{d}{d s} \log D(J ; \lambda)
$$

the third-order equation

$$
\frac{1}{2}\left(\frac{R^{\prime \prime}}{R^{\prime}}\right)^{\prime}-\frac{R}{R^{\prime}}+R^{\prime}=0
$$


It is also the case that

$$
R^{\prime}(s)=-q(s ; \lambda)^{2}
$$

and this gives the following simple formula for $D(J ; \lambda)$ in terms of a $\mathrm{P}_{I I}$ transcendent:

$$
D(J ; \lambda)=\exp \left(-\int_{s}^{\infty}(x-s) q(x ; \lambda)^{2} d x\right)
$$

This is much simpler than the corresponding representation of $D(J ; \lambda)$ for the sine kernel in terms of a $\mathrm{P}_{V}$ transcendent. The fact that $q(s ; \lambda)$ satisfies (1.11) can also be obtained by combining some results in [1, 5, 13]. Thus in this case of a semi-infinite interval our results connect with inverse scattering.

\section{Asymptotics and the Commuting Differential Operator}

Again we take $J=(s, \infty)$ and consider asymptotics as $s \rightarrow-\infty$. (Asymptotics as $s \rightarrow \infty$ can be obtained trivially from the Neumann series for $(I-K)^{-1}$.) From the random matrix point of view the interesting quantities are

$$
E(n ; s):=\left.\frac{(-1)^{n}}{n !} \frac{\partial^{n}}{\partial \lambda^{n}} D(I ; \lambda)\right|_{\lambda=1}
$$

This is the probability that exactly $n$ eigenvalues lie in $J$. Using our differential equations plus the known asymptotics of $q(s ; 1)$ as $s \rightarrow-\infty$ [13] we obtain the asymptotic expansions

$$
R(s ; 1) \sim \frac{1}{4} s^{2}-\frac{1}{8 s}+\frac{9}{64 s^{4}}-\frac{189}{128 s^{7}}+\frac{21663}{512 s^{10}}+\cdots \quad(s \rightarrow-\infty) .
$$

(where $R(s ; 1)$ denotes $R(s)$ corresponding to $\lambda=1$ ) and

$$
\begin{aligned}
E(0 ; s)= & D(J ; 1) \sim \frac{\tau_{0}}{(-s)^{1 / 8}} \exp \left(s^{3} / 12\right) \\
& \times\left(1-\frac{3}{2^{6} s^{3}}+\frac{2025}{2^{13} s^{6}}-\frac{2470825}{2^{19} s^{9}}+\cdots\right) \quad(s \rightarrow-\infty)
\end{aligned}
$$

where $\tau_{0}$ is an undetermined constant.

The analogue of this formula for the sine kernel was obtained by Dyson [8]. The analogue of our constant $\tau_{0}$ in the expansion was found to be equal to $2^{1 / 12} e^{3 \zeta^{\prime}(-1)}(\zeta(s)$ is the 
Riemann zeta function). This constant was obtained by scaling a result of the second author [30] on Toeplitz matrices. Such a result is not available to us now. Using (1.16) and the fact that $R(s)$ is the logarithmic derivative of $E(0 ; s)$, it is straightforward to derive an integral representation for $\log \tau_{0}$. This integral can be evaluated approximately by numerically integrating (1.11) and using ([1.14). This leads to the value $\log \tau_{0} \simeq-0.136540$. We conjecture that in fact

$$
\tau_{0}=e^{\zeta^{\prime}(-1)} 2^{\frac{1}{24}}=0.872371414954127 \ldots
$$

This agrees with the numerically computed value, up to its degree of accuracy.

For asymptotics of $E(n ; s)$ for general $n$ we introduce

$$
r(n ; s):=\frac{E(n ; s)}{E(0 ; s)} .
$$

Successive differentiation of (1.11) with respect to $\lambda$, plus the known asymptotics of $q(s ; 1)$, allows us to find asymptotic expansions for the quantities

$$
q_{n}(s):=\left.\frac{\partial^{n} q}{\partial \lambda^{n}}\right|_{\lambda=1}
$$

(for the analogue in the sine kernel case see [2]); and these in turn can be used to find expansions for $r(n ; s)$. One drawback of this approach is that yet another undetermined constant factor enters the picture. (In [2,31] Toeplitz and Wiener-Hopf techniques, not available for the Airy kernel, fixed this constant.) Another drawback is that when one expresses the $r(n ; s)$ in terms of the $q_{n}(s)$ a large amount of cancellation takes place, with the result that even the first-order asymptotics of $r(n ; s)$ are out of reach by this method when $n$ is large.

There is, however, another approach (briefly indicated in [2], and with details in [28], for the sine kernel case). We have

$$
r(n ; s)=\sum_{i_{1}<\cdots<i_{n}} \frac{\lambda_{i_{1}} \cdots \lambda_{i_{n}}}{\left(1-\lambda_{i_{1}}\right) \cdots\left(1-\lambda_{i_{n}}\right)}
$$

where $\lambda_{0}>\lambda_{1}>\cdots$ are the eigenvalues of the integral operator $K$ (with $\lambda=1$ ). Now just as the operator with the sine kernel commutes with the differential operator for the prolate 
spheroidal wave functions [14], so does the Airy operator commutes with the differential operator $L$ given by

$$
L f(x)=\left((x-s) f^{\prime}(x)\right)^{\prime}-x(x-s) f(x) .
$$

An application of the WKB method, plus a trick allows us to derive the following asymptotic formula for $\lambda_{i}$ with $i$ fixed:

$$
1-\lambda_{i} \sim \frac{\sqrt{\pi}}{i !} 2^{5 i+3} t^{3 i / 2+3 / 4} \exp \left(-\frac{8}{3} t^{3 / 2}\right) \quad(s=-2 t \rightarrow-\infty)
$$

(The analogue of this for the sine kernel is in [11.) From this and (1.19) we deduce

$$
r(n,-2 t) \sim \frac{1 ! 2 ! \cdots(n-1) !}{\pi^{n / 2} 2^{\left(5 n^{2}+n\right) / 2}} t^{-3 n^{2} / 4} \exp \left(\frac{8}{3} n t^{3 / 2}\right) .
$$

(Note that this can be used to fix the constant mentioned in the last paragraph.)

\section{E. Probability Density for the $n^{\text {th }}$ Largest Eigenvalue}

An elementary probability argument shows that if $F(n ; s)(n=0,1, \cdots)$ denotes the probability density for the (scaled) position of the $n^{\text {th }}$ largest eigenvalue $(n=0$ is the largest), then

$$
F(0 ; s)=\frac{d E(0 ; s)}{d s}
$$

and for $n \geq 1$

$$
F(n ; s)-F(n-1, s)=\frac{d E(n ; s)}{d s} .
$$

From the preceding expressions for $E(n ; s)$ in terms of $q(s ; 1)$ and $q_{n}(s)$ we can derive formulas for $F(n ; s)$ which can in turn be numerically evaluated to produce tables of values for these probability densities. We have done this for $n=0$ and $n=1$ and in Fig. 1 we plot $E(0 ; s)$ and $E(1 ; s)$ and in Fig. 2 we plot $F(0 ; s)$ and $F(1 ; s)$. 


\section{THE SYSTEM OF PARTIAL DIFFERENTIAL EQUATIONS}

\section{A. Derivation of the Equations}

A central role in the derivation of our equations is played by the commutator identity

$$
\left[L,(I-K)^{-1}\right]=(I-K)^{-1}[L, K](I-K)^{-1}
$$

for any operators $K$ and $L$. Taking $L$ to be $D(=d / d x)$ or $M$ (=multiplication by the independent variable) give two of the three basic ingredients from which the equations are derived. The third is the equally simple fact that if $K$ depends upon a parameter $\alpha$ then

$$
\frac{d}{d \alpha}(I-K)^{-1}=-(I-K)^{-1} \frac{d K}{d \alpha}(I-K)^{-1}
$$

It will be very useful for us to think of $K$ as acting not on $J$ but on $(-\infty, \infty)$ and to have kernel

$$
K(x, y) \chi_{J}(y)
$$

where $\chi_{J}$ is the characteristic function of $J$. We continue to denote the resolvent kernel of $K$ by $R(x, y)$ but note that while it is smooth in $x$ it is discontinuous at $y=a_{j}$. Thus the quantity $R\left(a_{j}, a_{j}\right)$ appearing in (1.1) must be interpreted to mean

$$
\lim _{\substack{y \rightarrow a_{j} \\ y \in J}} R\left(a_{j}, y\right)
$$

similarly for $p_{j}$ and $q_{j}$ in (1.2) and like quantities appearing later. The definitions of $u$ and $v$ must be modified to read

$$
u=\left(A \chi_{J},(I-K)^{-1} A\right), \quad v=\left(A \chi_{J},(I-K)^{-1} A^{\prime}\right)
$$

Notice that since

$$
(I-K)^{-1} A=(I-K)^{-1} A \chi_{J} \quad \text { in } J
$$

this agrees with the original definitions of $u$ and $v$ given by (1.3). 
We can think of $K$ as an operator taking smooth functions to smooth functions and so the operators $K D$ and $D K$ make sense, the former having distributional kernel. The transpose operator $K^{t}$ takes distributions to distributions.

With these preliminaries out of the way we begin with the derivation of the representations of the quantities $R\left(a_{j}, a_{k}\right)$ in terms of the $q_{j}, p_{j}, u$ and $v$. We introduce the notation

$$
Q=(I-K)^{-1} A, \quad P=(I-K)^{-1} A^{\prime}
$$

so that $q_{j}=Q\left(a_{j}\right), p_{j}=P\left(a_{j}\right)$. (For the moment we ignore the dependence of $Q$ and $P$ on the parameter $a=\left(a_{1}, \cdots, a_{2 m}\right)$; this will come later.)

The first commutator relation we use is the simple

$$
[M, K] \doteq\left(A(x) A^{\prime}(y)-A^{\prime}(x) A(y)\right) \chi_{J}(y)
$$

(where $\doteq$ means "has kernel equal to"). Hence from (2.1) with $L=M$

$$
\left[M,(I-K)^{-1}\right] \doteq Q(x)\left(I-K^{t}\right)^{-1} A^{\prime} \chi_{J}(y)-P(x)(I-K)^{-1} A \chi_{J}(y)
$$

(The transpose here arises from the general fact that if $L \doteq U(x) V(y)$, then $T_{1} L T_{2} \doteq$ $T_{1} U(x) T_{2}^{t} V(y)$.) If we use the fact that

$$
\left(I-K^{t}\right)^{-1} A \chi_{J}=(I-K)^{-1} A \text { on } J,
$$

we deduce from (2.5) and (2.6) that 15

$$
R(x, y)=\frac{Q(x) P(y)-P(x) Q(y)}{x-y} \quad(x, y \in J, x \neq y) .
$$

In particular, therefore,

$$
\begin{array}{r}
R\left(a_{j}, a_{k}\right)=\frac{q_{j} p_{k}-p_{j} q_{k}}{a_{j}-a_{k}} \quad(j \neq k), \\
R\left(a_{j}, a_{j}\right)=Q^{\prime}\left(a_{j}\right) p_{j}-P^{\prime}\left(a_{j}\right) q_{j} \quad\left({ }^{\prime}=\frac{d}{d x}\right) .
\end{array}
$$

To compute $Q^{\prime}(x)$ and $P^{\prime}(x)$ we consider first the commutator $[D, K]$. If an operator $L$ has distributional kernel $L(x, y)$ then 


$$
[D, L] \doteq\left(\frac{\partial}{\partial x}+\frac{\partial}{\partial y}\right) L(x, y)
$$

It is an easy verification, using the equation $A^{\prime \prime}(x)=x A(x)$, that

$$
\left(\frac{\partial}{\partial x}+\frac{\partial}{\partial y}\right) K(x, y)=-A(x) A(y)
$$

and so (recall the definition (2.3) of the kernel of $K$ )

$$
[D, K] \doteq-A(x) A(y) \chi_{J}(y)-\sum_{k}(-1)^{k} K\left(x, a_{k}\right) \delta\left(y-a_{k}\right) .
$$

Hence, applying (2.1) with $L=D$ (and recalling definitions (2.5)),

$$
\left[D,(I-K)^{-1}\right]=-Q(x)\left(I-K^{t}\right) A \chi_{J}(y)-\sum_{k}(-1)^{k} R\left(x, a_{k}\right) \rho\left(a_{k}, y\right)
$$

where

$$
\rho(x, y)=\delta(x-y)+R(x, y)
$$

is the distributional kernel of $(I-K)^{-1}$.

We proceed with the computations of $Q^{\prime}(x)$ and $P^{\prime}(x)$. Differentiating the first relation in (2.5) and using (2.13) give

$$
\begin{aligned}
Q^{\prime} & =D(I-K)^{-1} A=P+\left[D,(I-K)^{-1}\right] A \\
& =P-Q\left(\left(I-K^{t}\right)^{-1} A \chi_{J}, A\right)-\sum_{k}(-1)^{k} R\left(\cdot, a_{k}\right) q_{k} .
\end{aligned}
$$

Thus (recall (2.4))

$$
Q^{\prime}(x)=P(x)-Q(x) u-\sum_{k}(-1)^{k} R\left(x, a_{k}\right) q_{k} .
$$

Similarly

$$
P^{\prime}=(I-K)^{-1} A^{\prime \prime}+\left[D,(I-K)^{-1}\right] A^{\prime} .
$$

Since $A^{\prime \prime}(x)=x A(x)$ this is equal to

$$
M(I-K)^{-1} A-\left[M,(I-K)^{-1}\right] A+\left[D,(I-K)^{-1}\right] A^{\prime} .
$$


We now use formula (2.6) and (2.13) to deduce that

$$
P^{\prime}(x)=x Q(x)-2 Q(x) v+P(x) u-\sum_{k}(-1)^{k} R\left(x, a_{k}\right) p_{k} .
$$

Setting $x=a_{j}$ in (2.14) and (2.15) and using (2.10) give

$$
R\left(a_{j}, a_{j}\right)=p_{j}^{2}-a_{j} q_{j}^{2}+2 q_{j}^{2} v-2 p_{j} q_{j} u+\sum_{k}(-1)^{k} R\left(a_{j}, a_{k}\right)\left(q_{j} p_{k}-p_{j} q_{k}\right) .
$$

In view of (2.9) this is precisely equation (1.10).

Most of the work is already done and we can derive the equations (1.4)-(1.9) very quickly. First, we have the easy fact

$$
\frac{\partial}{\partial a_{k}} K \doteq(-1)^{k} K\left(x, a_{k}\right) \delta\left(y-a_{k}\right)
$$

and so by $(2.2)$

$$
\frac{\partial}{\partial a_{k}}(I-K)^{-1} \doteq(-1)^{k} R\left(x, a_{k}\right) \rho\left(y-a_{k}\right)
$$

At this point we must keep in mind that $Q$ and $P$ are functions of $a$ as well, and so we denote them now by $Q(x, a)$ and $P(x, a)$, respectively. We deduce immediately from (2.16) and the definitions (2.5) that

$$
\frac{\partial}{\partial a_{k}} Q(x, a)=(-1)^{k} R\left(x, a_{k}\right) q_{k}, \quad \frac{\partial}{\partial a_{k}} P(x, a)=(-1)^{k} R\left(x, a_{k}\right) p_{k} .
$$

Since $q_{j}=Q\left(a_{j}, a\right)$ and $p_{j}=P\left(a_{j}, a\right)$ this gives

$$
\frac{\partial q_{j}}{\partial a_{k}}=(-1)^{k} R\left(a_{j}, a_{k}\right) q_{k}, \quad \frac{\partial p_{j}}{\partial a_{k}}=(-1)^{k} R\left(a_{j}, a_{k}\right) p_{k} \quad(j \neq k) .
$$

In view of (2.9) these are equations (1.4) and (1.5). Moreover

$$
\frac{\partial q_{j}}{\partial a_{j}}=\left.\left(\frac{\partial}{\partial x}+\frac{\partial}{\partial a_{j}}\right) Q(x, a)\right|_{x=a_{j}}, \quad \frac{\partial p_{j}}{\partial a_{j}}=\left.\left(\frac{\partial}{\partial x}+\frac{\partial}{\partial a_{j}}\right) P(x, a)\right|_{x=a_{j}}
$$

and (2.17), (2.14) and (2.15) give

$$
\begin{aligned}
& \frac{\partial q_{j}}{\partial a_{j}}=p_{j}-q_{j} u-\sum_{k \neq j}(-1)^{k} R\left(a_{j}, a_{k}\right) q_{k}, \\
& \frac{\partial p_{j}}{\partial a_{j}}=a_{j} q_{j}-2 q_{j} v+p_{j} u-\sum_{k \neq j}(-1)^{k} R\left(a_{j}, a_{k}\right) p_{k} .
\end{aligned}
$$


In view of (2.9) again, these are equations (1.6) and (1.7).

Finally, using the definition of $u$ in (2.4), the fact

$$
\frac{\partial}{\partial a_{j}} \chi_{J}(y)=(-1)^{j} \delta\left(y-a_{j}\right)
$$

and (2.16) we find that

$$
\frac{\partial u}{\partial a_{j}}=(-1)^{j} A\left(a_{j}\right) q_{j}+(-1)^{j}\left(A \chi_{J}, R\left(\cdot, a_{j}\right)\right) q_{j}
$$

But

$$
\left(A \chi_{J}, R\left(\cdot, a_{j}\right)\right)=\int_{J} R\left(x, a_{j}\right) A(x) d x=\int_{J} R\left(a_{j}, x\right) A(x) d x
$$

since $R(x, y)=R(y, x)$ for $x, y \in J$. Since $R(y, x)=0$ for $x \notin J$ the last integral equals

$$
\int_{-\infty}^{\infty} R\left(a_{j}, x\right) A(x) d x=A\left(a_{j}\right)-q_{j}
$$

Thus

$$
\frac{\partial u}{\partial a_{j}}=(-1)^{j} q_{j}^{2}
$$

which is (1.8). Equation (1.9) is derived analogously.

We end this section with two relations between $u, v$, the $q_{j}$ and the $p_{j}$ which in fact can be used to represent $u$ and $v$ directly in terms of the $q_{j}$ and $p_{j}$. These relations are

$$
\begin{aligned}
2 v-u^{2} & =\sum_{j}(-1)^{j} q_{j}^{2}, \\
u & =-\sum_{j}\left(p_{j}^{2}-a_{j} q_{j}^{2}-2 p_{j} q_{j} u+2 q_{j}^{2} v\right) .
\end{aligned}
$$

To obtain the first of these observe that (1.4) and (1.6) imply

$$
\left(\sum_{k} \frac{\partial}{\partial a_{k}}\right) q_{j}=p_{j}-q_{j} u
$$

while from (1.8) and (1.9)

$$
\frac{\partial}{\partial a_{j}}\left(2 v-u^{2}\right)=2(-1)^{j} q_{j}\left(p_{j}-q_{j} u\right) .
$$


Hence if we multiply both sides of $(2.20)$ by $2(-1)^{j} q_{j}$ and sum over $j$ we obtain

$$
\left(\sum_{k} \frac{\partial}{\partial a_{k}}\right)\left(\sum_{j}(-1)^{j} q_{j}^{2}\right)=\left(\sum_{k} \frac{\partial}{\partial a_{k}}\right)\left(2 v-u^{2}\right) .
$$

It follows that the two sides of (2.18) differ by a function of $\left(a_{1}, \cdots, a_{2 m}\right)$ which is invariant under translation by any vector $(s, \cdots, s)$. Since, clearly, both sides tend to zero as all $a_{i} \rightarrow \infty$, their difference must be identically zero.

To deduce (2.19) we add (2.13) and (2.16) to obtain

$$
\left(\frac{\partial}{\partial x}+\frac{\partial}{\partial y}+\sum_{k} \frac{\partial}{\partial a_{k}}\right) R(x, y)=-Q(x) Q(y) \quad(x, y \in J) .
$$

(Observe that the kernel of $\left[D,(I-K)^{-1}\right]$ is $\left.(\partial / \partial x+\partial / \partial y) R(x, y).\right)$ Hence

$$
\left(\sum_{k} \frac{\partial}{\partial a_{k}}\right) R\left(a_{j}, a_{j}\right)=-q_{j}^{2}=-(-1)^{j} \frac{\partial u}{\partial a_{j}},
$$

the last by (1.8). Multiplying by $(-1)^{j}$ and summing over $j$ gives

$$
\left(\sum_{k} \frac{\partial}{\partial a_{k}}\right)\left(\sum_{j}(-1)^{j} R\left(a_{j}, a_{j}\right)\right)=-\left(\sum_{k} \frac{\partial}{\partial a_{k}}\right) u .
$$

From this we deduce, by an argument similar to the one at the end of the last paragraph, that

$$
\sum_{j}(-1)^{j} R\left(a_{j}, a_{j}\right)=-u
$$

If we substitute formula $(1.10)$ into this we see that the resulting double sum vanishes and we are left with (2.19).

\section{B. Hamiltonian Structure}

The partial differential equations (1.4)-(1.9) can be rewritten in a compact form that reveals a symplectic structure with (11.10) defining a family of commuting Hamiltonians. To see this we first change the notation slightly

$$
\begin{aligned}
q_{2 j} & =-\frac{i}{2} x_{2 j}, \quad p_{2 j}=-i y_{2 j}, \\
q_{2 j-1} & =\frac{1}{2} x_{2 j-1}, \quad p_{2 j-1}=y_{2 j-1},
\end{aligned}
$$


for $j=1,2, \ldots, m$, and

$$
v=\frac{1}{2} x_{0}, \quad u=y_{0}
$$

and introduce the canonical symplectic structure

$$
\left\{x_{j}, x_{k}\right\}=\left\{y_{j}, y_{k}\right\}=0, \quad\left\{x_{j}, y_{k}\right\}=\delta_{j k},
$$

for $j, k=0,1, \ldots, m$. Then equations (1.4)-(1.9) become

$$
d_{a} x_{j}=\left\{x_{j}, \omega(a)\right\} \quad \text { and } \quad d_{a} y_{j}=\left\{y_{j}, \omega(a)\right\} \quad(j=0,1, \ldots, m)
$$

where

$$
\begin{aligned}
\omega(a) & =d_{a} \log \operatorname{det}(I-K) \\
& =\sum_{j=1}^{2 m} G_{j}(x, y) d a_{j}
\end{aligned}
$$

and

$$
G_{j}(x, y)=y_{j}^{2}-\frac{1}{4} a_{j} x_{j}^{2}-x_{j} y_{j} y_{0}+\frac{1}{4} x_{j}^{2} x_{0}-\frac{1}{4} \sum_{\substack{k=1 \\ k \neq j}}^{2 m} \frac{\left(x_{j} y_{k}-x_{k} y_{j}\right)^{2}}{a_{j}-a_{k}}
$$

Furthermore, the $G_{j}$ 's are in involution

$$
\left\{G_{j}, G_{k}\right\}=0
$$

This last result can be verified by a direct calculation, but as we discuss below, there is a better way to understand why such Hamiltonians are in involution. We can summarize the system of equations by saying, in words, that to find the $a_{k}$-flow of the coordinates $x_{j}$ and $y_{j}$, one flows according to Hamilton's equation with Hamiltonian $G_{k}$. The consistency of these equations (i.e. mixed partials are equal) follows immediately from this Hamiltonian formulation (see, e.g. 28]).

When equations (1.4)-(1.9) are expressed in the Hamiltonian form (2.22), the equations are exactly analogous to the case of the sine kernel [17] and in both cases the Hamiltonians are defined via (2.23). For the JMMS equations (see, e.g. [28]) this Hamiltonian system is 
closely related to the Hamiltonian system studied by Moser 24]. In fact in both cases the systems of partial differential equations can be formulated in terms of the classical $R$-matrix approach to completely integrable systems. Such an approach identitfies the Lax operator and hence the commuting flow result above follows without any calculations. We refer the reader to [12 for a discussion of this classical $R$-matrix formulation of these systems of partial differential equations.

\section{THE ORDINARY DIFFERENTIAL EQUATIONS}

In this section we specialize to the case $J=(s, \infty)$ and derive the differential equations (1.11), (1.13) and (1.14). In the notation of the last section $m=1, a_{1}=s, a_{2}=\infty$. All

terms in the formulas we derived which contain $a_{2}$ vanish because of the decay of the Airy kernel at $+\infty$. Recall that we now denote the quantity $q$ corresponding to the endpoint $a_{1}=s$ by $q(s)$, and write $R(s)$ for $R(s, s)$.

First, equation (1.14) is immediate from (2.21), which now says that $R=u$, and (1.8).

For (1.13) we observe that (1.6) and (1.7) in the present case read

$$
\begin{aligned}
& q^{\prime}=p-q u, \\
& p^{\prime}=s q+p u-2 q v,
\end{aligned}
$$

whereas (1.10) reads

$$
R=p q^{\prime}-q p^{\prime}
$$

Thus, by (1.14),

$$
\frac{R}{R^{\prime}}=\left(\frac{p}{q}\right)^{\prime} .
$$

Taking the logarithmic derivative of both sides of (1.14) gives

$$
\frac{1}{2} \frac{R^{\prime \prime}}{R^{\prime}}=\frac{q^{\prime}}{q}
$$

and by (3.1) this equals 


$$
\frac{p}{q}-u=\frac{p}{q}-R
$$

Thus

$$
\frac{1}{2}\left(\frac{R^{\prime \prime}}{R^{\prime}}\right)^{\prime}=\left(\frac{p}{q}\right)^{\prime}-R^{\prime}
$$

By (3.3) this is just (1.13).

Finally, to obtain (1.11) we differentiate (3.1) and use (3.1), (3.2), (1.8) and (1.9). We find by an easy computation that

$$
q^{\prime \prime}=s q+q^{3}+q\left(u^{2}-2 v\right) .
$$

Conveniently, we have (2.18), which says in this case that $u^{2}-2 v=q^{2}$. Thus (1.11) is established.

\section{ASYMPTOTICS}

\section{A. Asymptotics via Painlevé}

Hastings and McLeod [13] (see also [5,6]) have shown that $q(s ; 1)$, the unique solution to

equation (1.11) which is asymptotic to $\operatorname{Ai}(s)$ as $s \rightarrow+\infty$, is asymptotically equal to $\sqrt{-s / 2}$ as $s \rightarrow-\infty$. In fact there is a complete asymptotic expansion in decreasing powers of $-s$, beginnning with this term [19]. Successive terms are easily computed. This result and later ones, become a little easier to state if we write $s=-t / 2$ :

$$
q(-t / 2 ; 1)=\frac{1}{2} \sqrt{t}\left(1-\frac{1}{t^{3}}-\frac{73}{2 t^{6}}-\frac{10657}{2 t^{9}}-\frac{13912277}{8 t^{12}}+\mathrm{O}\left(\frac{1}{t^{15}}\right)\right) \quad(t \rightarrow+\infty)
$$

Squaring and integrating and using (1.14) we deduce

$$
R(s)=\frac{1}{4} s^{2}+c-\frac{1}{8 s}+\frac{9}{64 s^{4}}-\frac{189}{128 s^{7}}+\frac{21663}{512 s^{10}}+\mathrm{O}\left(\frac{1}{s^{13}}\right) \quad(s \rightarrow-\infty)
$$

where $c$ is a constant. Substituting this into (1.13) shows that $c=0$. Thus we obtain (1.16) and then, after integrating and exponentiating, (1.17). 
To obtain the asymptotic expansions of $q_{n}(s)$ in (1.18), we differentiate (1.11) with respect to $\lambda$ and set $\lambda=1$. We obtain

$$
q_{1}^{\prime \prime}-\left(s+6 q_{0}^{2}\right) q_{1}=0
$$

(where $q_{0}(s)=q(s ; 1)$ ). Using (4.1) we find two linearly independent solutions of this equation, one exponentially large at $-\infty$ and one exponentially small. Assuming the exponentially large one actually appears with a nonzero factor we obtain the asymptotic expansion

$$
q_{1}(-t / 2)=c_{1} \frac{\exp \left(\frac{1}{3} t^{3 / 2}\right)}{t^{1 / 4}}\left(1+\frac{17}{24 t^{3 / 2}}+\frac{1513}{2^{7} 3^{2} t^{3}}+\frac{850193}{2^{10} 3^{4} t^{9 / 2}}+\frac{407117521}{2^{15} 3^{5} t^{6}}+\cdots\right)
$$

where $c_{1}$ is a constant factor to be determined.

Successive differentiations of (1.11) with respect to $\lambda$, followed by setting $\lambda=1$, yields a sequence of linear differential equations for the $q_{n}$,

$$
q_{n}^{\prime \prime}-\left(s+6 q_{0}^{2}\right) q_{n}=\varphi_{n}\left(q_{0}, \cdots, q_{n-1}\right),
$$

where $\varphi_{n}$ is a polynomial in the $q_{m}$ with $m<n$. For $n>1$ a particular solution dominates all solutions of the homogeneous equation. Therefore, in the asymptotics, no new unknown constant factors are introduced (unless we want to go "beyond all orders").

To deduce expansions for the $r(n ; s)$ we must differentiate (1.15) $n$ times with respect to $\lambda$ and set $\lambda=1$. We find expressions of the form

$$
r(n ; s)=\int_{s}^{\infty}(x-s) \psi\left(q_{0}, \cdots, q_{n}\right) d x
$$

where $\psi_{n}$ is a polynomial in $q_{0}, \cdots, q_{n}$. In particular we obtain (after setting $s=-t / 2$ )

$$
r(1 ;-t / 2)=\frac{1}{2} \int_{-\infty}^{t}(t-x) q_{0}(-x / 2) q_{1}(-x / 2) d x
$$

Substituting (4.1) and (4.2) into this integral gives the asymptotic expansion for $r(1 ;-t / 2)$. The first order result is

$$
r(1 ;-t / 2) \sim c_{1} \frac{\exp \left(t^{3 / 2} / 3\right)}{t^{3 / 4}}
$$


and comparing this with $(1.22)$ reveals that

$$
c_{1}=\frac{1}{2 \sqrt{2 \pi}}
$$

We now state the expansions we have obtained in this way with the help of Mathematica. For $q_{n}$ with $n>2$, as $t \rightarrow+\infty$

$$
\begin{aligned}
q_{n}(-t / 2)= & \frac{n !}{2^{3 n / 2} \pi^{n / 2}} \frac{\exp \left(\frac{n}{3} t^{3 / 2}\right)}{t^{3 n / 4-1 / 2}}\left(1+\frac{59 n-36}{24} t^{-3 / 2}+\right. \\
& \left.\frac{3481 n^{2}+8244 n-8496}{1152} t^{-3}+\mathrm{O}\left(t^{-9 / 2}\right)\right) .
\end{aligned}
$$

For $n=1,2$ the leading order term is as above. For $n=1$ the correction terms are given above in (4.2). For $n=2$ the coefficient of $t^{-3 / 2}$ is as given above but the coefficient of $t^{-3}$ is $5461 / 288$. (Similar exceptional values of $n$ occurred in analogous expansions for the sine kernel [2].) These expansions have been computed for $n \leq 10$ and are undoubtedly correct for all $n$. (In [2] the analogous expansions were proved for all $n$ by a backwards induction argument - such arguments should extend to the present case of $q_{n}$.)

For $r(n ; s)$ we have obtained the following sharpening of (1.22): as $t \rightarrow+\infty$

$$
\begin{aligned}
r(n ;-t / 2)=\frac{1 ! 2 ! \cdots(n-1) !}{2^{n^{2}+n / 2} \pi^{n / 2}} \frac{\exp \left(\frac{n}{3} t^{3 / 2}\right)}{t^{3 n^{2} / 4}}\left(1+\frac{n\left(34 n^{2}+31\right)}{24} t^{-3 / 2}+\right. \\
\\
\left.\frac{n^{2}\left(1156 n^{4}+6608 n^{2}+11509\right)}{1152} t^{-3}+\mathrm{O}\left(t^{-9 / 2}\right)\right) .
\end{aligned}
$$

This was established for $n \leq 6$. As was mentioned in the Introduction, when we substitute

expansions (4.4) into (4.3) a large amount of cancellation takes place, so that $r(n ; s)$ is much smaller than might be anticipated. This also means that to deduce even the first-order asymptotics in (4.5) for moderate values of $n$ one has to go very far out in the expansions (4.4). Thus it is important that we have an alternative approach which yields asymptotic results for all values of $n$.

\section{B. Asymptotics via the Commuting Differential Operator}

We begin with the integral identity 


$$
K(x, y)=\int_{0}^{\infty} A(x+z) A(y+z) d z
$$

This appears in [5] but we give a simple proof here. Think of (2.12) as a differential equation for the "unknown" function $K(x, y)$. Since

$$
\left(\frac{\partial}{\partial x}+\frac{\partial}{\partial y}\right) A(x+z) A(y+z)=\frac{\partial}{\partial z} A(x+z) A(y+z)
$$

one solution is the right side of (4.6) and, of course, another solution is the Airy kernel $K(x, y)$. The difference is therefore of the form $\varphi(x-y)$ for some function $\varphi$. Since both sides of (4.6) tend to zero as $x$ and $y$ tend to $+\infty$ independently, we must have $\varphi \equiv 0$.

Rewriting (4.6) as

$$
K(x, y)=\int_{s}^{\infty} A(x+z-s) A(z+y-s) d z
$$

shows that $K$ as an operator on $(s, \infty)$ is the square of the operator with kernel $A(x+y-s)$.

Integration by parts shows that an integral operator with kernel $L(x, y)$ on $(s, \infty)$ commutes with a differential operator

$$
\frac{d}{d x} \alpha(x) \frac{d}{d x}+\beta(x)
$$

if $\alpha(s)=0$ and if

$$
\alpha(y) \frac{\partial^{2} L}{\partial y^{2}}+\alpha^{\prime}(y) \frac{\partial L}{\partial y}+\beta(y) L=\alpha(x) \frac{\partial^{2} L}{\partial x^{2}}+\alpha^{\prime}(x) \frac{\partial L}{\partial x}+\beta(x) L .
$$

(Of course we also require, in the end, appropriate vanishing at $\infty$.) If we set $L(x, y)=$ $A(x+y-s)$ and use the fact $A^{\prime \prime}(x)=x A(x)$ we can easily check that a solution is given by

$$
\alpha(x)=x-s, \quad \beta(x)=-x(x-s)
$$

and so we obtain the commuting differential operator (1.20).

Here is an outline of how (1.21) is found. If we let $f_{1}, f_{2}, \cdots$ denote the eigenfunctions of $L$ then the logarithmic derivative of each $\lambda_{i}$ (thought of as a function of $s$ ) is expressible very simply in terms of the corresponding $f_{i}$. The asymptotics of these eigenfunctions, and so of the logarithmic derivative, is established by WKB techniques. Integrating, and using 
the fact that each $\lambda_{i} \rightarrow 1$ as $s \rightarrow-\infty$, gives (1.21). The WKB argument we give is quite heuristic although it could very likely be made rigorous.

We make a preliminary change of variables, replacing $x$ by $x+s$ (so that $L$ acts on $(0, \infty))$ and $s$ by $-2 t$. The eigenvalue problem can then be written as

$$
\left(x f^{\prime}(x)\right)^{\prime}+\left(\mu-(x-t)^{2}\right) f(x)=0
$$

or in the equivalent form

$$
x g^{\prime \prime}(x)+\left[\mu-(x-t)^{2}+\frac{1}{4 x}\right] g(x)=0, \quad(g(x)=\sqrt{x} f(x))
$$

The eigenvalues satisfy $0<\mu_{0}<\mu_{1}<\cdots$ and oscillation considerations applied to (4.8) show that $\mu_{i}=\mathrm{O}\left(t^{1 / 2}\right)$, for each $i$ as $t \rightarrow \infty$. So we think of $\mu$ in our equations as $\mathrm{O}\left(t^{1 / 2}\right)$, we normalize our eigenfunctions so that $f(0)=1$, and proceed to find asymptotics for large $t$.

\section{The region $x<<t^{-1 / 2}$}

If we make the substitutions

$$
f(x)=h\left(t^{2} x\right)=h(y)
$$

then (4.7) becomes

$$
\left(y h^{\prime}(y)\right)^{\prime}-h(y)=t^{-2}\left(\frac{y^{2}}{t^{4}}-2 \frac{y}{t}-\mu\right) h(y) .
$$

The solution of the equation with the right hand side replaced by 0 which satisfies $h(0)=1$ is $I_{0}(2 \sqrt{y})$ where $I_{0}$ is the usual modified Bessel function. The actual solution $h(y)$ will be asymptotic to this in any interval over which the integral of the factor of $h(y)$ on the right hand side of the equation is o(1). Recalling that $\mu=\mathrm{O}\left(t^{1 / 2}\right)$ we see that $y<<t^{3 / 2}$ suffices. Hence

$$
f(x) \sim I_{0}(2 t \sqrt{x}), \quad\left(x<<t^{-1 / 2}\right)
$$




\section{The region $x>>t^{-2}, t-x>>t^{1 / 4}$}

If in equation (4.8) we set

$$
\alpha(x)=\frac{(t-x)^{2}-\mu}{x}, \quad y=\int_{0}^{x} \sqrt{\alpha(z)} d z, \quad h(y)=\alpha(x)^{1 / 4} g(x)
$$

then the equation for $h(y)$ is

$$
h^{\prime \prime}(y)-h(y)=\left[\frac{1}{4} \frac{\alpha^{\prime \prime}}{\alpha^{2}}-\frac{5}{16} \frac{\alpha^{\prime 2}}{\alpha^{3}}-\frac{1}{4 x^{2} \alpha}\right] h(y)
$$

where on the right side $^{\prime}=\frac{d}{d x}$. Since $d y=\alpha(x)^{1 / 2} d x$, the integral with respect to $y$ (of the factor of $h(y)$ on the right side of the equation) over any interval equals

$$
\int\left(\frac{1}{4} \frac{\alpha^{\prime \prime}}{\alpha^{3 / 2}}-\frac{5}{16} \frac{\alpha^{2}}{\alpha^{5 / 2}}-\frac{1}{4 x^{2} \alpha^{1 / 2}}\right) d x
$$

over the corresponding $x$ interval. This will be o(1) as long as, in this interval, $x>>t^{-2}$ and $t-x>>t^{1 / 4}$.

In this same region $y>>1$ and so

$$
h(y) \sim a e^{y}
$$

for some constant $a=a(t)$. In case $x<<1$ we have $y=2 t \sqrt{x}+\mathrm{o}(1)$ and so

$$
f(x)=x^{-1 / 2} g(x) \sim a t^{-1 / 2} x^{-1 / 4} e^{2 t \sqrt{x}}, \quad\left(t^{-2}<<x<<1\right) .
$$

Comparing this result with (4.9) in the overlapping region $t^{-2}<<x<<t^{-1 / 2}$ and using the known asymptotics of $I_{0}(z)$ as $z \rightarrow \infty$ shows that $a \sim 1 / 2 \sqrt{\pi}$. Hence from (4.10)

$$
f(x) \sim \frac{1}{2 \sqrt{\pi}} \frac{(t-x)^{-1 / 2}}{x^{1 / 4}} \exp \left\{\int_{0}^{x} \sqrt{\frac{(t-z)^{2}-\mu}{z}} d z\right\}, \quad\left(x>>t^{-2}, t-x>>t^{1 / 4}\right) .
$$

We also used here once again the estimate $\mu=\mathrm{O}\left(t^{1 / 2}\right)$. Because of this same estimate we can write the integral in the exponential as

$$
\int_{0}^{x} \frac{t-z}{\sqrt{z}}\left\{1-\frac{1}{2} \frac{\mu}{(t-z)^{2}}+\mathrm{O}\left(\frac{\mu^{2}}{(t-z)^{4}}\right)\right\} d z
$$


Because $t-x>>t^{1 / 4}$ the last term in brackets contributes o(1) to the integral, and so we obtain

$$
2 t x^{1 / 2}-\frac{2}{3} x^{3 / 2}-\frac{\mu}{2} \int_{0}^{x} \frac{d z}{\sqrt{z}(t-z)} .
$$

Replacing $z$ by $t z^{2}$ shows that the integral here equals

$$
t^{-1 / 2} \log \frac{(\sqrt{t}+\sqrt{x})^{2}}{t-x} .
$$

Thus

$$
\begin{aligned}
& f(x) \sim \frac{1}{2 \sqrt{\pi}} \frac{(t-x)^{\frac{1}{2} t^{-1 / 2} \mu-\frac{1}{2}}}{x^{1 / 4}(\sqrt{t}+\sqrt{x})^{t^{-1 / 2} \mu}} \exp \left\{2 t x^{1 / 2}-\frac{2}{3} x^{3 / 2}\right\} \\
&\left(x>>t^{-2}, t-x>>t^{1 / 4}\right) .
\end{aligned}
$$

Let us see what happens if $x$ is not far from $t$, if

$$
t^{1 / 4}<<t-x<<t^{1 / 2} .
$$

Then since

$$
2 t x^{1 / 2}-\frac{2}{3} x^{3 / 2}=\frac{4}{3} t^{4 / 3}-\frac{1}{2}(x-t)^{2}+o(1)
$$

in this region, we find

$$
\begin{aligned}
f(x) \sim \frac{1}{2 \sqrt{\pi}} 2^{-t^{-1 / 2} \mu} t^{-3 / 4}\left(\frac{t}{t-x}\right)^{-\frac{1}{2} t^{-1 / 2} \mu+\frac{1}{2}} \exp \left\{\frac{4}{3} t^{4 / 3}-\frac{1}{2}(x-t)^{2}\right\} \\
\left(t^{1 / 4}<<t-x<<t^{1 / 2}\right)
\end{aligned}
$$

\section{The region $x-t>>t^{1 / 4}$}

This is quite similar to the preceding. The main difference is that instead of making the variable change $y=\int \alpha(x)^{1 / 2} d x$ in (4.8) we now set

$$
y=\frac{2}{3} x^{3 / 2}-2 t x^{1 / 2}-\int_{x}^{\infty}\left[\sqrt{\alpha(z)}-z^{1 / 2}+t z^{-1 / 2}\right] d z .
$$


Since an eigenfunction cannot grow exponentially at $\infty$ only the negative exponential $e^{-y}$ can appear in the asymptotics and we find that

$$
f(x) \sim b \frac{(x-t)^{-1 / 2}}{x^{1 / 4}} \exp \left\{2 t x^{1 / 2}-\frac{2}{3} x^{3 / 2}+\int_{x}^{\infty}\left[\sqrt{\frac{(z-t)^{2}-\mu}{z}}-z^{1 / 2}+t z^{-1 / 2}\right] d z\right\}
$$

for some constant $b=b(t)$. Now the integral in the exponential is

$$
-\frac{\mu}{2} t^{-1 / 2} \log \frac{(\sqrt{x}+\sqrt{t})^{2}}{x-t}+\mathrm{o}(1)
$$

and so

$$
f(x) \sim b \frac{(x-t)^{\frac{1}{2} t^{-1 / 2} \mu-\frac{1}{2}}}{x^{1 / 4}(\sqrt{x}+\sqrt{t})^{t^{-1 / 2} \mu}} \exp \left\{2 t x^{1 / 2}-\frac{2}{3} x^{3 / 2}\right\} \quad\left(x-t>>t^{1 / 4}\right)
$$

and specializing to the region $t^{1 / 4}<<x-t<<t^{1 / 2}$ gives

$$
\begin{aligned}
& f(x) \sim b 2^{-t^{-1 / 2} \mu} t^{-3 / 4}\left(\frac{t}{x-t}\right)^{-\frac{1}{2} t^{-1 / 2} \mu+\frac{1}{2}} \exp \left\{\frac{4}{3} t^{3 / 2}-\frac{1}{2}(x-t)^{2}\right\} \\
&\left(t^{1 / 4}<<x-t<<t^{1 / 2}\right)
\end{aligned}
$$

4. The region $|x-t|<<t$

If in equation (4.8) we set

$$
g(x)=h\left(\sqrt{2} t^{-1 / 4}(t-x)\right)=h(y)
$$

and write $\varepsilon=\left(\sqrt{2} t^{3 / 4}\right)^{-1}$ then the equation becomes

$$
(1-\varepsilon y) h^{\prime \prime}(y)+\left[\frac{\mu}{2 t^{1 / 2}}-\frac{y^{2}}{4}+\frac{\varepsilon^{2}}{4(1-\varepsilon y)}\right] h(y)=0 .
$$

Now we expect that for bounded $y$, and therefore also if $|y| \rightarrow \infty$ at some rate (depending upon $\varepsilon$ ), the solution of this will be asymptotic to a solution of

$$
h^{\prime \prime}(y)+\left(\frac{\mu}{2 t^{1 / 2}}-\frac{y^{2}}{4}\right) h(y)=0
$$

Weber's equation. Now unless 


$$
\frac{\mu}{2 t^{1 / 2}}=i+\frac{1}{2} \quad(i=0,1, \cdots)
$$

there is no solution which vanishes at both $\pm \infty$. But (4.12) and (4.14) show that in their regions of validity (which overlap with our present region) $f(x)$ is a factor depending on $t$ times a factor tending exponentially to 0 as $x-t \rightarrow \pm \infty$. Hence our eigenvalues must satisfy

$$
\frac{\mu_{i}}{2 t^{1 / 2}}=i+\frac{1}{2}+\mathrm{o}(1)
$$

and $h(y)$ is asymptotically a constant times the parabolic cylinder function $D_{i}(y)$ (see, e.g. [4], Chp. 8). Thus (since $x \sim t$ in the present situation)

$$
f(x) \sim c D_{i}\left(\sqrt{2} t^{-1 / 4}(t-x)\right)
$$

for some constant $c=c(t)$. Using the known asymptotics of $D_{i}$ we find

$$
f(x) \sim c\left(\sqrt{2} t^{-1 / 4}(t-x)\right)^{i} \exp \left\{-\frac{1}{2} t^{-1 / 2}(t-x)^{2}\right\}
$$

Comparing this with (4.12) gives

$$
c \sim \frac{2^{-\frac{5}{2} i-2}}{\sqrt{\pi}} t^{-\frac{3}{4} i-\frac{3}{4}} \exp \left(\frac{4}{3} t^{3 / 2}\right) .
$$

We also find, using (4.14), that $b \sim 1 / 2 \sqrt{\pi}$.

\section{The asymptotics of $\lambda_{i}$}

If we look at the asymptotics of $f_{i}(x)$ (the eigenfunction associated with the eigenvalue $\mu_{i}$ of $L$, normalized so that $\left.f_{i}(0)=1\right)$ given by $(4.9),(4.11),(4.13)$, and (4.16) we see that the main contribution to $\int_{0}^{\infty} f_{i}(x)^{2} d x$ comes from any region

$$
t^{-1 / 4}|t-x|<\eta(t)
$$

as long as $\eta(t) \rightarrow \infty$. For some such region we have, by (4.15) and (4.17),

$$
f_{i}(x) \sim \frac{2^{-\frac{5}{2} i-2}}{\sqrt{\pi}} t^{-\frac{3}{4} i-\frac{3}{4}} D_{i}\left(\sqrt{2} t^{-1 / 4}(t-x)\right) .
$$


Using this, and the fact $\int_{-\infty}^{\infty} D_{i}(x) d x=\sqrt{2 \pi} i$, we deduce

$$
\int_{0}^{\infty} f_{i}(x)^{2} d x \sim \frac{i !}{\sqrt{\pi}} 2^{-5 i-4} t^{-\frac{3}{2} i-\frac{5}{4}} \exp \left(\frac{8}{3} t^{3 / 2}\right)
$$

This was the goal of all that went before.

Since $L$ has simple eigenvalues and commutes with our integral operator $K$ (translated to $(0, \infty))$ the set $\left\{f_{i}\right\}$ is the set of eigenfunctions of $K$ corresponding to its eigenvalues $\left\{\lambda_{i}\right\}$. However $f_{i}$ corresponds to $\mu_{i}$ and there is no assurance that the corresponding eigenvalue of $K$ is its $i^{\text {th }}$ largest, which we have denoted by $\lambda_{i}$. We shall eventually show that this is actually the case. The first lemma of this section shows that the eigenvalues of $K$ are simple, and this is crucial for the argument we shall later use. (The proof of the corresponding result for the sine kernel is in [27].)

Lemma 1 If $f_{1}$ and $f_{2}$ are eigenfunctions of $L$ corresponding to distinct eigenvalues, then they are eigenfunctions of $K$ corresponding to distinct eigenvalues.

Proof. The eigenvalues of $K$ are the squares of the eigenvalues of the operator on $(0, \infty)$ with kernel $A(x+y-2 t)$. So we have to show that if for some $\nu$ we have either

$$
\int_{0}^{\infty} A(x+y-2 t) f_{i}(y) d y=\nu f_{i}(x) \quad(i=1,2)
$$

or

$$
\int_{0}^{\infty} A(x+y-2 t) f_{1}(y)=\nu f_{1}(x), \quad \int_{0}^{\infty} A(x+y-2 t) f_{2}(y) d y=-\nu f_{2}(x)
$$

then $f_{1}=f_{2}$. (Recall that all eigenfunctions are normalized to satisfy $f(0)=1$.)

Assuming first that (4.19) holds, apply $d^{2} / d x^{2}$ to both sides of the identity with $i=1$ and integrate the resulting integral by parts twice. What results is

$$
\nu f_{1}^{\prime \prime}(x)=-A^{\prime}(x-2 t)+A(x-2 t) f_{1}^{\prime}(0)+\int_{0}^{\infty} A(x+y-2 t) f_{1}^{\prime \prime}(y) d y
$$

If we multiply both sides by $f_{2}(x)$ and integrate we obtain, using (4.19) with $i=2$ (and its differentiated version), 


$$
\nu \int_{0}^{\infty} f_{1}^{\prime \prime}(x) f_{2}(x) d x=-\nu f_{2}^{\prime}(0)+\nu f_{1}^{\prime}(0)+\nu \int_{0}^{\infty} f_{2}(y) f_{1}^{\prime \prime}(y) d y
$$

Thus, since $\nu \neq 0$ (this follows easily from the fact that the Fourier transform of $A(x)$ is nonzero), we have $f_{1}^{\prime}(0)=f_{2}^{\prime}(0)$. But it is clear from (4.7) that for any eigenfunction $f$ we have $f^{\prime}(0)=t^{2}-\mu$. Thus $f_{1}$ and $f_{2}$ correspond to the same eigenvalue of $L$ and so are equal.

The case when (4.20) holds is even easier. Differentiating both sides of the first relation once and integrating by parts gives

$$
\nu f_{1}^{\prime}(x)=-A(x-2 t)-\int_{0}^{\infty} A(x+y-2 t) f_{1}^{\prime}(y) d y
$$

Multiplying both sides of this by $f_{2}(x)$ and integrating, using the second relation of (4.20), we obtain

$$
\nu \int_{0}^{\infty} f_{1}^{\prime}(x) f_{2}(x) d x=-\nu+\nu \int_{0}^{\infty} f_{2}(y) f_{1}^{\prime}(y) d y
$$

contradicting $\nu \neq 0$.

Since, as we now know, the eigenvalues of $K$ are simple, there is a permutation $\sigma$ of $\mathbf{N}=\{0,1,2, \cdots\}$ such that $f_{i}$ is the eigenfunction of $K$ corresponding to its $\sigma(i)^{t h}$ largest eigenvalue $\lambda_{\sigma(i)}$; this permutation is independent of $t$ or else, by the continuity of the eigenvalues in $t$, there would be a multiple eigenvalue for some $t$.

Up to now our kernel $K$ corresponded to an arbitrary factor $\lambda$. Of course formula (1.21) refers to the case $\lambda=1$.

Lemma 2 For each $i$ we have $\lambda_{i} \rightarrow 1$ as $s \rightarrow-\infty$.

Proof. In this case $\lambda=1$ formula (4.6) reads

$$
K(x, y)=\int_{0}^{\infty} \operatorname{Ai}(x+z) \operatorname{Ai}(z+y) d z
$$

For the purposes of this proof we denote by $A$ (resp. $K$ ) the operator on $(-\infty, \infty)$ with kernel $\mathrm{Ai}(x+y)$ (resp. $K(x, y))$ and by $P_{s}$ the projection from $L_{2}(-\infty, \infty)$ to $L_{2}(s, \infty)$. So the $\lambda_{i}$ are the eigenvalues of $P_{s} K P_{s}$. Now it is known [13] that $A^{2}=I$, and (4.21) says that $K=A P_{0} A$. Thus $K^{2}=A P_{0} A^{2} P_{0} A=A P_{0} A=K$. In other words, $K$ is a projection 
operator. (This is not surprising since its kernel is a scaling limit of the kernels $K_{N}(x, y)$ of projection operators.) Clearly it has infinite-dimensional range. It now follows easily from the minimax characterization of the eigenvalues that, for each $i$, the $i^{\text {th }}$ largest eigenvalue of $P_{s} K P_{s}$ tends to 1 as $s \rightarrow-\infty$.

We now state a lemma which is the basis of a trick used by Fuchs 11 in his derivation of the asymptotics of the eigenvalues of the sine kernel.

Lemma 3 Let $J(x, y)$ be a symmetric kernel, on a fixed interval, depending smoothly on a parameter $t$. Let $g$ be an eigenfunction normalized so that $\int g(x)^{2} d x=1$ and let $\lambda$ be the corresponding eigenvalue. Then, with the subscript $t$ denoting $\partial / \partial t$, we have

$$
\lambda_{t}=\iint J_{t}(x, y) g(y) g(x) d y d x
$$

Proof. From

$$
\int J(x, y) g(y) d y=\lambda g(x)
$$

we get

$$
\int J_{t}(x, y) g(y) d y+\int J(x, y) g_{t}(y) d y=\lambda_{t} g(x)+\lambda g_{t}(x)
$$

Multiplying both sides by $g(x)$ and integrating over $x$, we obtain (using the normalization of $g$ and (4.22)

$$
\begin{aligned}
\iint J_{t}(x, y) g(y) g(x) d y d x+\iint J(x, y) g_{t}(y) g(x) d x d y & =\lambda_{t}+\lambda \int g_{t}(x) g(x) d x \\
& =\lambda_{t}+\iint J(x, y) g(y) g_{t}(x) d y d x
\end{aligned}
$$

By symmetry of $J$ the two double integrals involving it cancel, and we obtain the statement of the lemma.

We apply the lemma to the opertor with kernel $A(x+y-2 t)$ on $(0, \infty)$ whose square is our Airy operator (translated to act on $(0, \infty)$ ). Thus we may write its eigenvalues as $\sqrt{\lambda_{\sigma(i)}}$, where the square roots might have either sign. The lemma gives 


$$
\frac{\partial}{\partial t} \sqrt{\lambda_{\sigma(i)}}=-2 \int_{0}^{\infty} \int_{0}^{\infty} A^{\prime}(x+y-2 t) g_{i}(y) g_{i}(x) d y d x
$$

where

$$
g_{i}(x)=f_{i}(x) /\left\{\int_{0}^{\infty} f_{i}(y)^{2} d y\right\}^{1 / 2}
$$

But from

$$
\int_{0}^{\infty} A(x+y-2 t) g_{i}(y) d y=\sqrt{\lambda_{\sigma(i)}} g_{i}(x)
$$

we obtain upon differentiation that

$$
\int_{0}^{\infty} A^{\prime}(x+y-2 t) g_{i}(y) d y=\sqrt{\lambda_{\sigma(i)}} g_{i}^{\prime}(x)
$$

so (4.23) may be rewritten

$$
\frac{\partial}{\partial t} \sqrt{\lambda_{\sigma(i)}}=-2 \sqrt{\lambda_{\sigma(i)}} \int_{0}^{\infty} g_{i}^{\prime}(x) g_{i}(x) d x .
$$

The integral is of course $-\frac{1}{2} g_{i}(0)^{2}$. Recalling that $f_{i}(0)=1$ we see that we have established the relation

$$
\frac{\partial}{\partial t} \sqrt{\lambda_{\sigma(i)}}=\sqrt{\lambda_{\sigma(i)}} /\left\{\int_{0}^{\infty} f_{i}(x)^{2} d x\right\}
$$

or

$$
\frac{\partial}{\partial t} \log \lambda_{\sigma(i)}=2 /\left\{\int_{0}^{\infty} f_{i}(x)^{2} d x\right\}
$$

We now use (4.18) and integrate the resulting relation with respect to $t$ from $t$ to $\infty$. Recalling Lemma 2, we deduce

$$
-\log \lambda_{\sigma(i)} \sim \frac{\sqrt{\pi}}{i !} 2^{5 i+3} t^{\frac{3}{2} i+\frac{3}{4}} \exp \left(-\frac{8}{3} t^{3 / 2}\right)
$$

precisely the right side of (1.21).

All that remains now is to show that $\sigma(i)=i$ for all $i$. But it is clear from this asymptotic relation that $i<j$ implies $\lambda_{\sigma(i)}>\lambda_{\sigma(j)}$ for large $t$ (and so for all $t$ ), so that $\sigma(i)<\sigma(j)$. Thus $\sigma$ is order preserving, and since $\sigma: \mathbf{N} \rightarrow \mathbf{N}$ is onto we must have $\sigma(i)=i$ for all $i$.

This concludes the derivation of ([1.21). The deduction of (1.22) from (1.21) is completely analogous to the corresponding deduction for the sine kernel in ref. [28], Sec. VIIB. 


\section{ACKNOWLEDGMENTS}

We wish to thank E. Brézin, P. J. Forrester, F. A. Grünbaum and J. Harnad for helpful comments. This work was supported in part by the National Science Foundation, DMS9001794 and DMS-9216203, and this support is gratefully acknowledged. 


\section{REFERENCES}

[1] M. J. Ablowitz and H. Segur, Exact linearization of a Painlevé transcendent, Phys. Rev. Letts. 38 (1977) 1103-1106.

[2] E. L. Basor, C. A. Tracy, and H. Widom, Asymptotics of level spacing distributions for random matrices, Phys. Rev. Letts. 69 (1992) 5-8.

[3] M. J. Bowick and E. Brézin, Universal scaling of the tail of the density of eigenvalues in random matrix models, Phys. Letts. B268 (1991) 21-28.

[4] A. Erdélyi (ed.), Higher Transcendental Functions, Vol. II (McGraw-Hill, New York, 1953).

[5] P. A. Clarkson and J. B. McLeod, A connection formula for the second Painlevé transcendent, Arch. Rat. Mech. Anal. 103 (1988) 97-138.

[6] P. A. Clarkson and J. B. McLeod, Integral equations and connection formulae for the Painlevé equations, in Painlevé Transcendents: Their Asymptotics and Physical Applications, eds. D. Levi and P. Winternitz (Plenum Press, New York, 1992), pgs. 1-31.

[7] F. J. Dyson, Statistical theory of energy levels of complex systems, I, II, and III, J. Math. Phys. 3 (1962) 140-156; 157-165; 166-175.

[8] F. J. Dyson, Fredholm determinants and inverse scattering problems, Commun. Math. Phys. 47 (1976) 171-183.

[9] F. J. Dyson, The Coulomb fluid and the fifth Painlevé transcendent, IASSNSS-HEP92/43 preprint, to appear in the proceedings of a conference in honor of C. N. Yang, ed. S.-T. Yau.

[10] P. J. Forrester, The spectrum edge of random matrix ensembles, preprint.

[11] W. H. J. Fuchs, On the eigenvalues of an integral equation arising in the theory of band-limited signals, J. Math. Anal. and Applic. 9 (1964) 317-330. 
[12] J. Harnad, C. A. Tracy, and H. Widom, Hamiltonian structure of equations appearing in random matrices, in preparation.

[13] S. P. Hastings and J. B. McLeod, A boundary value problem associated with the second Painlevé transcendent and the Korteweg-de Vries equation, Arch. Rat. Mech. Anal. 73 (1980) $31-51$.

[14] E. L. Ince, Ordinary Differential Equations, (Dover, New York, 1956).

[15] A. R. Its, A. G. Izergin, V. E. Korepin, and N. A. Slavnov, Differential equations for quantum correlation functions, Int. J. Mod. Physics B 4 (1990) 1003-1037.

[16] K. Iwasaki, H. Kimura, S. Shimomura, and M. Yoshida, From Gauss to Painlevé: A Modern Theory of Special Functions (Vieweg, Braunschweig, 1991).

[17] M. Jimbo, T. Miwa, Y. Môri, and M. Sato, Density matrix of an impenetrable Bose gas and the fifth Painlevé transcendent, Physica 1D (1980) 80-158.

[18] B. M. McCoy, C. A. Tracy, and T. T. Wu, Connection between the KdV equation and the two-dimensional Ising model, Phys. Letts. 61A (1977) 283-284.

[19] J. B. McLeod, private communication.

[20] M. L. Mehta, Random Matrices, 2nd edition (Academic, San Diego, 1991).

[21] M. L. Mehta, A non-linear differential equation and a Fredholm determinant, J. de Phys. I France, 2 (1992) 1721-1729.

[22] M. L. Mehta and G. Mahoux, Level spacing functions and non-linear differential equations, preprint.

[23] G. Moore, Matrix models of 2D gravity and isomonodromic deformation, Prog. Theor. Physics Suppl. No. 102 (1990) 255-285.

[24] J. Moser, Geometry of quadrics and spectral theory, in Chern Symposium 1979 
(Springer, Berlin, 1980), 147-188.

[25] P. Painlevé, Sur les équations différentielles du second ordre et d'ordre supérieur dont l'intégrale générale est uniforme, Acta Math. 25 (1902) 1-85.

[26] C. E. Porter, Statistical Theory of Spectra: Fluctuations (Academic, New York, 1965).

[27] D. Slepian and H. O. Pollak, Prolate spheroidal wave functions, Fourier analysis and uncertainty-I, Bell Systems Tech. J. 40 (1961) 43-64.

[28] C. A. Tracy and H. Widom, Introduction to random matrices, to appear in the proceedings of the $8^{\text {th }}$ Scheveningen Conference, Springer Lecture Notes in Physics.

[29] C. A. Tracy and H. Widom, Level spacing distributions and the Airy kernel, submitted to Phys. Letts. B.

[30] H. Widom, The strong Szegö limit theorem for circular arcs, Indiana Univ. Math. J. 21 (1971) 277-283.

[31] H. Widom, The asymptotics of a continuous analogue of orthogonal polynomials, to appear in J. Approx. Th. 


\section{FIGURES}

FIG. 1. The probabilities $E(0 ; s)$ and $E(1 ; s)$. Of course, $E(0 ; s) \rightarrow 1$ as $s \rightarrow+\infty$.

FIG. 2. The probability densities $F(0 ; s)$ and $F(1 ; s)$. The expected position of the largest eigenvalue is approximately -1.7711 with variance 0.8132 . The expected position of the next-largest eigenvalue is approximately -3.6754 with variance 0.5405 . 